\title{
A multicenter prospective cohort study of volume management after subarachnoid hemorrhage: circulatory characteristics of pulmonary edema after subarachnoid hemorrhage
}

\author{
*Yoshiki Obata, MD, ${ }^{1,6}$ Junichi Takeda, MD, ${ }^{2,6}$ Yohei Sato, MD, ${ }^{1,6}$ Hiroyasu Ishikura, MD, PhD, 3,6 \\ Toru Matsui, MD, DMSc, ${ }^{4,6}$ and Eiji Isotani, MD, PhD 5,6 \\ ${ }^{1}$ Department of Neurosurgery, Tokyo Medical and Dental University; ${ }^{2}$ Department of Neurosurgery, Kansai Medical University \\ Takii Hospital, Osaka; ${ }^{3}$ Department of Emergency and Critical Care Medicine, Fukuoka University Hospital, Fukuoka; \\ ${ }^{4}$ Department of Neurosurgery, Saitama Medical Center, Saitama; ${ }^{5}$ Emergency and Critical Care Center, Tokyo Women's Medical \\ University Medical Center East, Japan; and ${ }^{6} \mathrm{SAH}$ PiCCO Study Group
}

\begin{abstract}
OBJECTIVE Subarachnoid hemorrhage (SAH) is often accompanied by pulmonary complications, which may lead to poor outcomes and death. This study investigated the incidence and cause of pulmonary edema in patients with SAH by using hemodynamic monitoring with PiCCO-plus pulse contour analysis.

METHODS A total of 204 patients with SAH were included in a multicenter prospective cohort study to investigate hemodynamic changes after surgical clipping or coil embolization of ruptured cerebral aneurysms by using a PiCCO-plus device. Changes in various hemodynamic parameters after SAH were analyzed statistically.

RESULTS Fifty-two patients (25.5\%) developed pulmonary edema. Patients with pulmonary edema (PE group) were significantly older than those without pulmonary edema (non-PE group) $(p=0.017)$. The mean extravascular lung water index was significantly higher in the PE group than in the non-PE group throughout the study period. The pulmonary vascular permeability index (PVPI) was significantly higher in the PE group than in the non-PE group on Day $6(p=$ $0.029)$ and Day $10(p=0.011)$. The cardiac index of the PE group was significantly decreased biphasically on Days 2 and 10 compared with that of the non-PE group. In the early phase (Days 1-5 after SAH), the daily water balance of the PE group was slightly positive. In the delayed phase (Days 6-14 after SAH), the serum C-reactive protein level and the global end-diastolic volume index were significantly higher in the PE group than in the non-PE group, whereas the PVPI tended to be higher in the PE group.
\end{abstract}

CONCLUSIONS Pulmonary edema that occurs in the early and delayed phases after SAH is caused by cardiac failure and inflammatory (i.e., noncardiogenic) conditions, respectively. Measurement of the extravascular lung water index, cardiac index, and PVPI by PiCCO-plus monitoring is useful for identifying pulmonary edema in patients with SAH.

Clinical trial registration no.: UMIN000003794 (clinicaltrials.gov)

http://thejns.org/doi/abs/10.3171/2015.6.JNS1519

KEY WORDS subarachnoid hemorrhage; thermodilution; pulmonary edema; extravascular lung water; vascular permeability; acute respiratory distress syndrome; vascular disorders

$\mathrm{S}$ UBARACHNOID hemorrhage (SAH) from a ruptured aneurysm is accompanied by several complications, including electrolyte abnormalities, vasospasm, hydrocephalus, cardiac dysfunction, and pulmonary edema. ${ }^{15,16,18}$ Among these complications, delayed cerebral ischemia (DCI) remains an important cause of morbidity and death. ${ }^{15}$ Many studies have investigated the prevention or treatment of DCI after SAH.

Triple-H therapy (i.e., hypertension, hypervolemia, and hemodilution) has reportedly been an effective approach

ABBREVIATIONS $\mathrm{Cl}=$ cardiac index; $\mathrm{CRP}=\mathrm{C}$-reactive protein; $\mathrm{DCl}=$ delayed cerebral ischemia; ELWI = extravascular lung water index; $\mathrm{GEDI}=$ global end-diastolic volume index; PVPI = pulmonary vascular permeability index; $\mathrm{SAH}=$ subarachnoid hemorrhage; $\mathrm{SVRI}=$ systemic vascular resistance index; WFNS = World Federation of Neurosurgical Societies.

SUBMITTED January 6, 2015. ACCEPTED June 3, 2015.

INCLUDE WHEN CITING Published online November 27, 2015; DOI: 10.3171/2015.6.JNS1519.

* Drs. Obata and Takeda contributed equally to this work. 
for preventing DCI after SAH., ${ }^{2,17}$ In contrast, triple-H therapy has been shown to not be superior to normovolemic fluid therapy in the prevention of DCI. ${ }^{5}$ Moreover, Lennihan et al. ${ }^{19}$ have reported that triple-H therapy does not increase cerebral blood flow. Therefore, it is necessary to validate the benefits of triple- $\mathrm{H}$ therapy and clarify the characteristics of systemic circulation after SAH.

Volume management is critical in the treatment of patients with SAH. However, maintaining optimal fluid balance in these patients is sometimes difficult. Some studies have suggested that triple-H therapy increases the incidence of heart failure and pulmonary edema in patients with SAH. $5,19,38$ Pulmonary edema is common after SAH; neurogenic pulmonary edema may occur in the acute phase of SAH. Moreover, pulmonary edema associated with SAH has a variety of causes. Pulse contour analysis using a PiCCO-plus device (Pulsion Medical Systems) calibrated by transpulmonary thermodilution was used recently to monitor various parameters, including cardiac output, contractility, afterload, volume management, and lung water, in critically ill patients. ${ }^{23,30}$ Accordingly, in this multicenter prospective cohort study, we investigated postoperative changes in the cardiopulmonary circulation in patients with SAH by using the PiCCO-plus pulse contour device.

\section{Methods \\ Study Population}

A total of 204 patients with SAH were enrolled between October 2008 and April 2012 from 9 institutions in Japan. The patients or their families provided written informed consent before participation. The exclusion criteria were as follows: age $\leq 15$ years, disappearance of brainstem reflexes, cardiopulmonary dysfunction requiring percutaneous cardiopulmonary support, and pregnancy.

\section{Study Protocol}

For each patient, PiCCO-plus monitoring was started after surgery for SAH. A PiCCO catheter (Pulsion Medical Systems) was inserted into the brachial or femoral artery, and a central venous line was inserted into the superior vena cava. Both lines were connected to the PiCCO-plus device to continuously monitor cardiac output, contractility, and afterload. Transpulmonary thermodilution was performed to monitor volume management and lung water. To obtain measurements, we administered a $15-\mathrm{ml}$ bolus injection of ice-cold saline solution into the superior vena cava of each patient, and the thermistor of the PiCCO catheter was placed in the brachial or femoral artery. Transpulmonary thermodilution was performed 3 times/day, and the average value was stored in the SAH PiCCO databank.

\section{Parameters and Measurements}

The PiCCO-plus system calculates cardiac output from thermodilution curves according to the Stewart-Hamilton algorithm and the mean transit time and exponential downslope time of the thermodilution curve. The intrathoracic thermal volume was calculated as the product of cardiac output and mean transit time, and the pulmonary thermal volume was calculated as the product of cardiac output and exponential downslope time..$^{21}$ The global enddiastolic volume index (GEDI) was defined as the difference between the intrathoracic and pulmonary thermal volumes. ${ }^{25,31}$ The cardiac function index was defined as the ratio of cardiac output to the GEDI. The intrathoracic blood volume was calculated as GEDI $\times 1.25$. Extravascular lung water volume was calculated as the difference between the intrathoracic thermal volume and the intrathoracic blood volume. ${ }^{26}$ The pulmonary blood volume was calculated as the difference between the pulmonary thermal volume and extravascular lung water volume. The pulmonary vascular permeability index (PVPI) was defined as the ratio of the extravascular lung water volume to pulmonary blood volume.

The reference ranges for each parameter were as follows: cardiac index (CI) (cardiac output indexed to body surface area), 3.0-5.0 $\mathrm{L} \times \mathrm{min}^{-1} \times \mathrm{m}^{-2}$; systemic vascular resistance index (SVRI), 1700-2400 dynes $\times \mathrm{sec} \times \mathrm{cm}^{-5} \times$ $\mathrm{m}$; GEDI (indexed to body surface area), $680-800 \mathrm{ml} / \mathrm{m}^{2}$; intrathoracic blood volume (indexed to body surface area), $850-1000 \mathrm{ml} / \mathrm{m}^{2}$; extravascular lung water index (ELWI) (extravascular lung water volume indexed to body weight), $3.0-10.0 \mathrm{ml} / \mathrm{kg}$; and PVPI, 1.0-3.0.

The following data were stored in the SAH PiCCO databank: various PiCCO-plus measurements, World Federation of Neurosurgical Societies (WFNS) grade, ${ }^{37}$ Hunt and Kosnik grade, ${ }^{12}$ Fisher grade, ${ }^{6}$ aneurysm site, treatment, serum brain natriuretic peptide concentration for PiCCO catheter insertion (PiCCO on) and removal (PiCCO off), laboratory blood values from admission to Day 14 , daily water balance, central venous pressure, development of DCI, and the relationship between triple-H therapy and the incidence of pulmonary edema. Echocardiographic and electrocardiographic data were not stored in the SAH PiCCO databank. DCI was defined as a new neurological deterioration, either transient or permanent, in the absence of any other identifiable cause of neurological deterioration, including surgical complication, hydrocephalus, intracranial rebleeding, seizure, infection, and metabolic disturbance. ${ }^{34}$ Pulmonary edema was defined as an arterial oxygen partial pressure to fractional inspired oxygen ratio of $<300$ and evidence of bilateral infiltrative shadows on chest radiography. ${ }^{23}$

Patient outcomes were evaluated on Day 30 by using the Glasgow Outcome Scale. ${ }^{14}$ Morbidity was defined as any result other than satisfactory recovery.

\section{Statistical Analysis}

The chi-square test was used to analyze differences in the morbidity, mortality, and symptomatic vasospasm rates between patients with pulmonary edema (PE group) and those without pulmonary edema (non-PE group). The Mann-Whitney U-test was used to compare blood laboratory examination results, gross water balance, central venous pressure, and $\mathrm{PiCCO}$-plus measurements between the 2 groups. All statistical analyses were performed by using GraphPad Prism version 5.0 (GraphPad Software, Inc.), except for the multivariate regression analysis, which was performed by using JMP 11 (SAS Institute, Inc.). A $p$ value of $<0.05$ was set as the level of significance. 


\section{Results}

Of the 204 patients enrolled in the SAH PiCCO study (see Appendix for a list of participating centers), 52 patients $(25.5 \%)$ developed pulmonary edema (PE group). Table 1 shows the baseline characteristics of the patients. One patient with Hunt and Kosnik grade and Fisher grade data missing from the SAH PiCCO databank was excluded from the analysis. Patients in the PE group were significantly older than those in the non-PE group $(p=0.015)$. The mean serum brain natriuretic peptide level at the time of PiCCO catheter insertion was significantly higher in the PE group than in the non-PE group. Hunt and Kosnik grades, Fisher grades, and aneurysm locations were not significantly different between the PE and non-PE groups. Forty-four patients (21.6\%) developed DCI. Seventy-one patients $(34.8 \%)$ received triple-H therapy; 23 (32.4\%) of these patients developed pulmonary edema. The morbidity rates in the PE and non-PE groups were $63.5 \%$ and $47.4 \%$, respectively $(\mathrm{p}=0.054)$. The mortality rates in the $\mathrm{PE}$ and non-PE groups were $7.7 \%$ and $5.8 \%$, respectively $(\mathrm{p}=0.73)$.

Reliable data were obtained for 177 patients (49 in the
PE group and 128 in the non-PE group). Figure 1 shows the cardiac output, contractility, and afterload in both groups. The CI was significantly lower in the PE group than in the non-PE group on Day $2(\mathrm{p}=0.017)$ and Day $10(\mathrm{p}=0.022)$. The cardiac function index was significantly lower in the PE group than in the non-PE group on Day $10(p=0.039)$. The global ejection fraction was not significantly different between the groups. Compared with the non-PE group, the mean arterial pressure was significantly lower in the PE group on Day $5(\mathrm{p}=0.010)$, Day $7(\mathrm{p}=0.019)$, and Day $10(p=0.013)$. The SVRI was not significantly different between the groups.

Figure 2 shows the volume management and lung water content results according to PiCCO-plus monitoring. The ELWI was significantly higher in the PE group than in the non-PE group on Day $3(\mathrm{p}=0.019)$, Day $6(\mathrm{p}=0.0035)$, Day $7(\mathrm{p}=0.010)$, Day $8(\mathrm{p}=0.033)$, Day $10(\mathrm{p}=0.018)$, Day $11(\mathrm{p}=0.039)$, and Day $12(\mathrm{p}=0.027)$. Daily water balance was significantly different between the 2 groups on Day $1(p=0.027)$ and Day $2(p=0.027)$. Central venous pressure was significantly higher in the PE group than in the non-PE group on Day $3(\mathrm{p}=0.0051)$ and Day $11(p=0.038)$. The GEDI was not significantly different

TABLE 1. Characteristics of the study population

\begin{tabular}{|c|c|c|c|c|}
\hline Characteristic & All Patients & PE Group & Non-PE Group & $p$ Value \\
\hline No. of cases & 204 & 52 & 152 & \\
\hline Age (yrs) & $63.2 \pm 12.9$ & $67.0 \pm 12.2$ & $61.9 \pm 12.9$ & 0.015 \\
\hline $\operatorname{Sex}(M / F)$ & $65 / 139$ & $16 / 36$ & $49 / 103$ & 0.84 \\
\hline WFNS grade & & & & 0.37 \\
\hline I & 18 & 4 & 14 & \\
\hline II & 35 & 5 & 30 & \\
\hline III & 12 & 2 & 10 & \\
\hline IV & 49 & 14 & 35 & \\
\hline V & 89 & 27 & 62 & \\
\hline Fisher grade & & & & 0.23 \\
\hline 1 & 1 & 0 & 1 & \\
\hline 2 & 11 & 1 & 10 & \\
\hline 3 & 119 & 28 & 91 & \\
\hline 4 & 72 & 23 & 49 & \\
\hline Aneurysm site & & & & 0.20 \\
\hline $\mathrm{ACoA}$ & 58 & 19 & 39 & \\
\hline ICA & 57 & 16 & 41 & \\
\hline MCA & 61 & 10 & 51 & \\
\hline VA/BA & 28 & 7 & 21 & \\
\hline Treatment: clipping & 161 & 39 & 122 & 0.42 \\
\hline \multicolumn{5}{|l|}{ BNP level (pg/ml)* } \\
\hline PiCCO on & $202.2 \pm 161.5$ & $288.2 \pm 237.8$ & $151.3 \pm 110.3$ & 0.03 \\
\hline PiCCO off & $177.3 \pm 143.2$ & $263.5 \pm 215.7$ & $130.2 \pm 100.7$ & 0.20 \\
\hline Triple-H therapy & 71 & 23 & 48 & 0.12 \\
\hline $\mathrm{DCl}$ & 44 & 10 & 34 & 0.63 \\
\hline Morbidity rate (\%) & 52.0 & 63.5 & 48.0 & 0.054 \\
\hline Mortality rate (\%) & 6.4 & 7.7 & 5.8 & 0.73 \\
\hline
\end{tabular}

$\mathrm{ACOA}=$ anterior communicating artery; $\mathrm{BNP}=$ brain natriuretic peptide; $\mathrm{ICA}=$ internal carotid artery; $\mathrm{MCA}=$ middle cerebral artery; $V A / B A=$ vertebral artery/basilar artery.

* Values are presented as the mean \pm SD. 

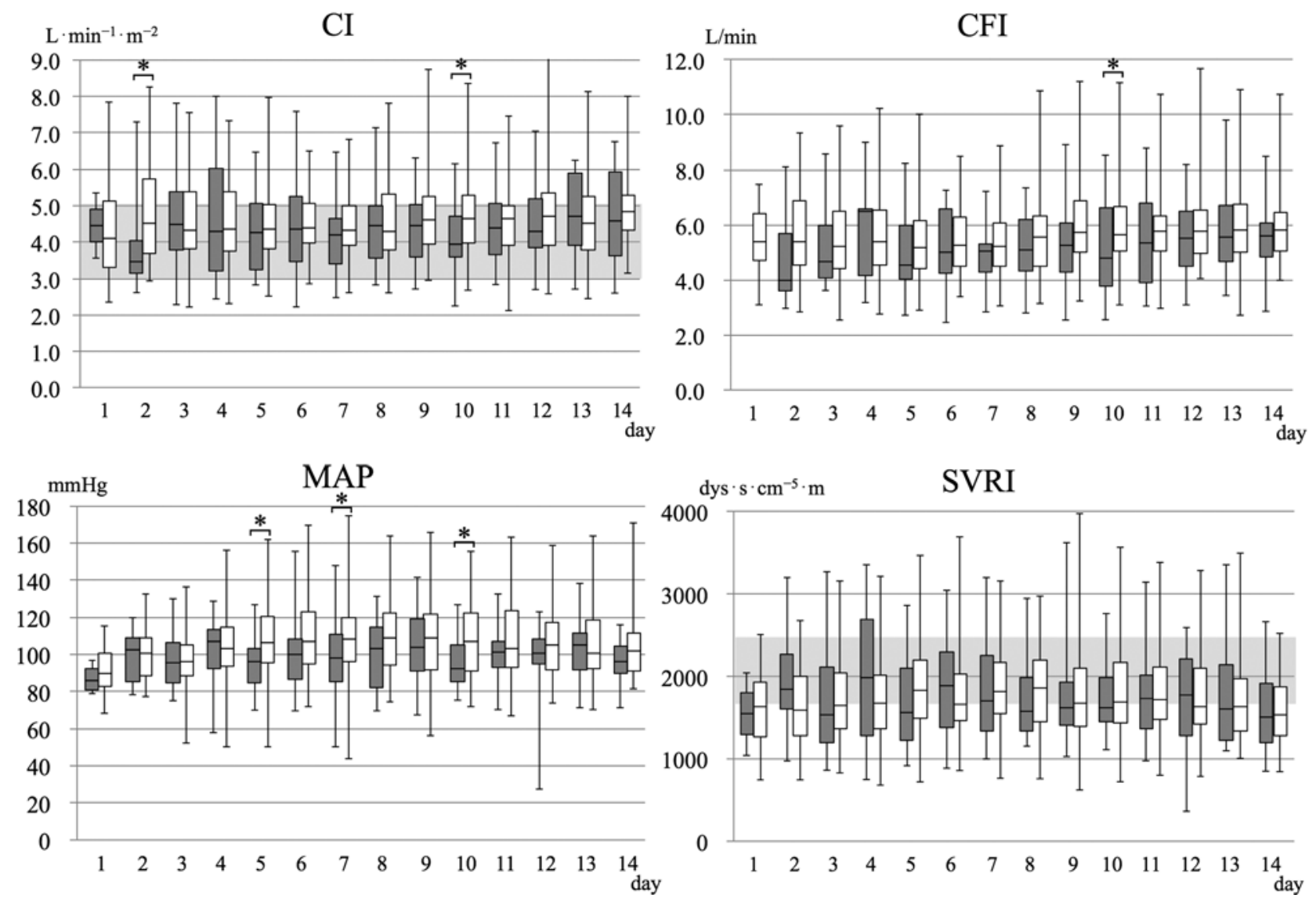

FIG. 1. PiCCO-plus monitoring after SAH: output, contractility, and afterload. Gray and white boxes represent data from the PE and non-PE groups, respectively; asterisks indicate statistical significance. $\mathrm{CFI}=$ cardiac function index; dys = dynes; MAP = mean arterial pressure.

between the 2 groups. The PVPI was significantly higher in the PE group than in the non-PE group on Day $6(\mathrm{p}=$ $0.029)$ and Day $10(\mathrm{p}=0.011)$.

The causes of pulmonary edema presumably differ according to the timing of onset after SAH. Therefore, pulmonary edema onset was classified as 1 of 2 phases: early or delayed. Horie et al. ${ }^{28}$ defined the vasospasm period as Days 6-8 after SAH. Therefore, Days 1-5 and 6-14 after SAH were defined as the early and delayed phases, respectively. Eighteen and 14 PE group patients developed pulmonary edema in the early and delayed phases, respectively. In addition, 19 patients developed pulmonary edema on Day 0 . PiCCO data from the onset of pulmonary edema in these patients were not recorded. Therefore, data from patients with pulmonary edema that developed on Day 0 were not included in the early-phase analyses.

Early-phase PiCCO data are shown in Figs. 3 and 4. The ELWI was significantly higher in the PE group than in the non-PE group on Day $3(\mathrm{p}=0.004)$, Day $4(\mathrm{p}=$ $0.039)$, Day $5(\mathrm{p}=0.016)$, Day $6(\mathrm{p}=0.0055)$, Day $7(\mathrm{p}=$ $0.0009)$, and Day $8(\mathrm{p}=0.037)$. The CI was significantly lower in the early-phase PE group than in the non-PE group on Day $2(\mathrm{p}=0.028)$. The cardiac function index was significantly lower in the early-phase PE group than in the non-PE group on Day $2(\mathrm{p}=0.042)$ and Day 3 ( $\mathrm{p}$ $=0.041)$. The global ejection fraction and the GEDI were not significantly different between the 2 groups. The mean arterial pressure and the SVRI were significantly lower in the PE group than in the non-PE group on Day $5(\mathrm{p}$ $=0.049$ and 0.030, respectively), Day $7(\mathrm{p}=0.020$ and 0.025 , respectively), and Day $8(\mathrm{p}=0.012$ and 0.016 , respectively). Central venous pressure was significantly higher in the PE group than in the non-PE group on Day 7 $(p=0.012)$. Compared with the non-PE group, daily water balance was significantly more positive in the early-phase PE group on Day $1(p=0.017)$ and Day $2(p=0.013)$. The PVPI was not significantly different between the 2 groups.

In the delayed phase, the ELWI $(\mathrm{p}=0.039)$, PVPI $(\mathrm{p}=$ $0.031)$, and daily water balance $(\mathrm{p}=0.025)$ were significantly higher in the PE group than in the non-PE group on Day 6. In addition, the PVPI tended to be higher in the PE group than in the non-PE group on Day $9(\mathrm{p}=0.054)$ and Day $10(\mathrm{p}=0.069)$. The CI, global ejection fraction, and SVRI were not significantly different between the groups. The GEDI was significantly higher in the PE group than in the non-PE group on Day $8(\mathrm{p}=0.0036)$ and Day $9(\mathrm{p}$ $=0.030$ ). Central venous pressure was significantly higher in the PE group than in the non-PE group on Day $11(\mathrm{p}=$ $0.005)$ and Day $12(p=0.023)$. The serum C-reactive protein (CRP) level was significantly higher in the PE group than in the non-PE group on Day $5(\mathrm{p}=0.046)$, Day 8 $(\mathrm{p}=0.021)$, and Day $9(\mathrm{p}=0.036)$. In contrast, neither 

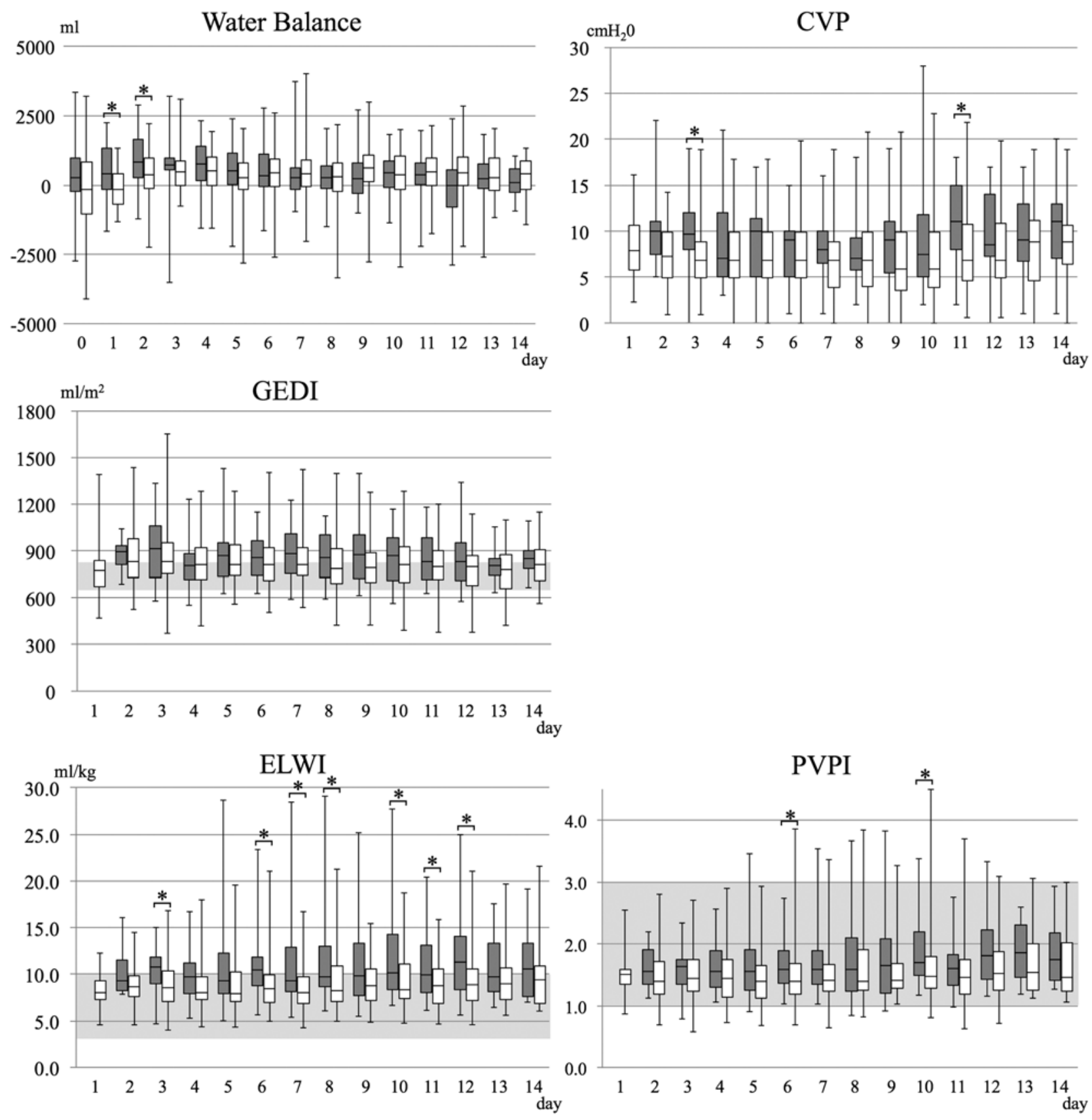

FIG. 2. PiCCO-plus monitoring after SAH: volume management and lung parameters. Gray and white boxes represent data from the PE and non-PE groups, respectively; asterisks indicate statistical significance. CVP = central venous pressure.

white blood cell count nor body temperature differed significantly between the groups (Figs. 5 and 6). Last, neither the ELWI nor the PVPI was associated with serum CRP levels, which reflect the degree of inflammation (Fig. 7). Multivariate analysis revealed that age, WFNS grade, and PVPI on Day 6 were independent risk factors for the occurrence of pulmonary edema in the delayed phase $(\mathrm{p}=$ $0.002,0.009$, and 0.024, respectively; Table 2).

The serum CRP levels in the delayed-phase PE and non-PE groups were highest on Day 3. Therefore, we analyzed the relationship between serum CRP level and ELWI on Day 3. The serum CRP level on Day 3 was posi- tively correlated with ELWI on Day 3 in the delayed-phase PE group (Fig. 8).

\section{Discussion}

Pulmonary edema is a critical complication after SAH that significantly increases the risk of morbidity and death. ${ }^{7}$ Recent studies indicated that $10 \%-29 \%$ of patients with SAH develop pulmonary edema.,11,24 Moreover, Horie et al. ${ }^{28}$ reported that surgical clipping can increase the incidence of pulmonary edema during the vasospasm period. Pulmonary edema worsens the prognosis of SAH 

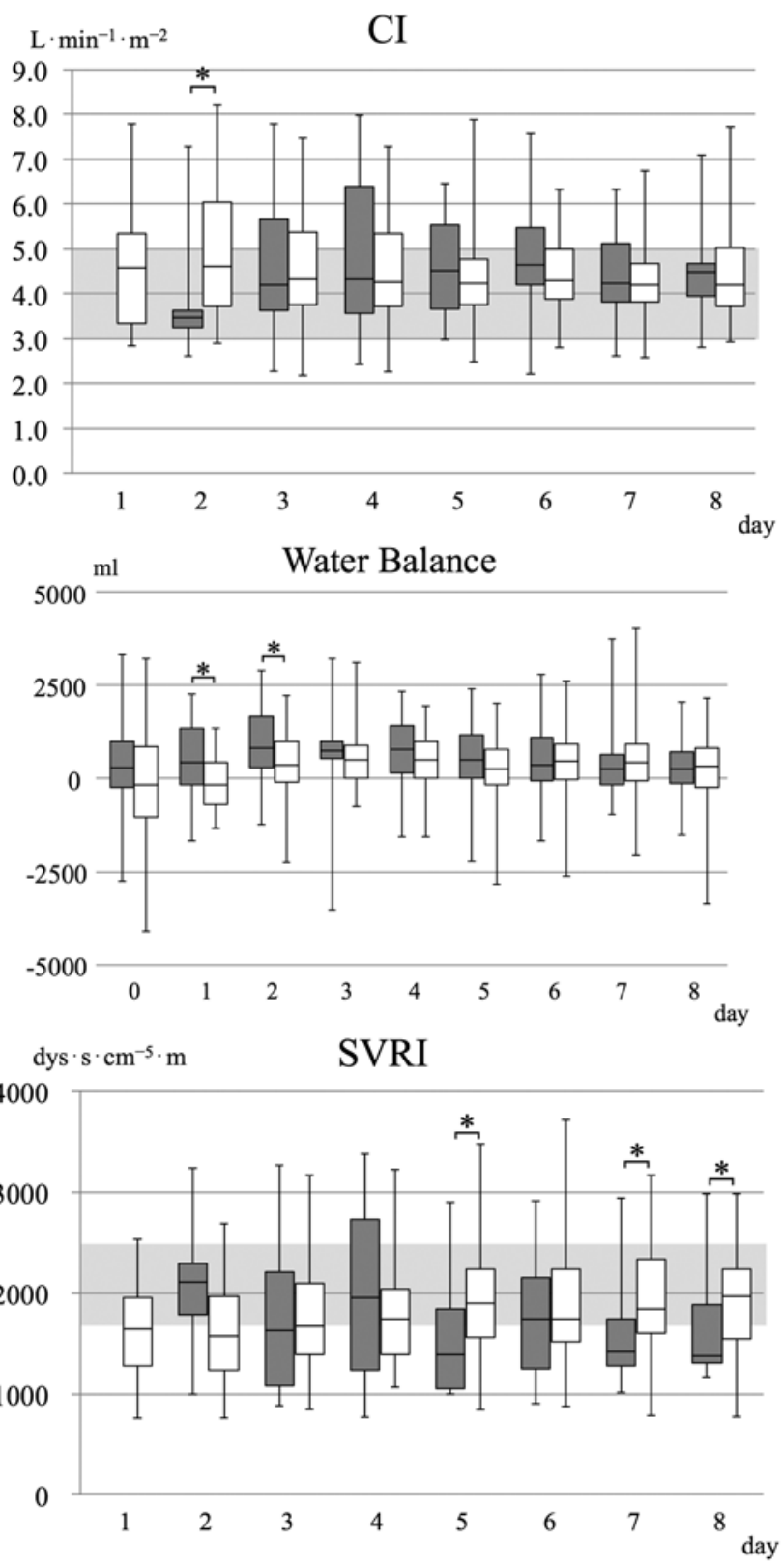

FIG. 3. PiCCO-plus monitoring in the early phase. Gray and white boxes represent data from the PE and non-PE groups, respectively; asterisks indicate statistical significance.

and increases the mortality rate. ${ }^{20}$ Therefore, preventing the development of pulmonary edema in patients with SAH is of utmost importance. In our study, the incidence of pulmonary edema in patients with SAH was $25 \%$, and the rate was higher in older patients. This finding is consistent with those of previous studies.7 The PE group had a worse SAH grade than the non-PE group ${ }^{7,10}$ Regarding radiological findings, severe bleeding is observed more often in patients with pulmonary edema. ${ }^{24}$ In addition, pulmonary edema is biphasic depending on the aneurysm location (i.e., anterior or middle cerebral artery aneurysm in the acute phase or internal carotid artery aneurysm in the spasm phase). ${ }^{27}$ In our study, clinical grade, Fisher grade,
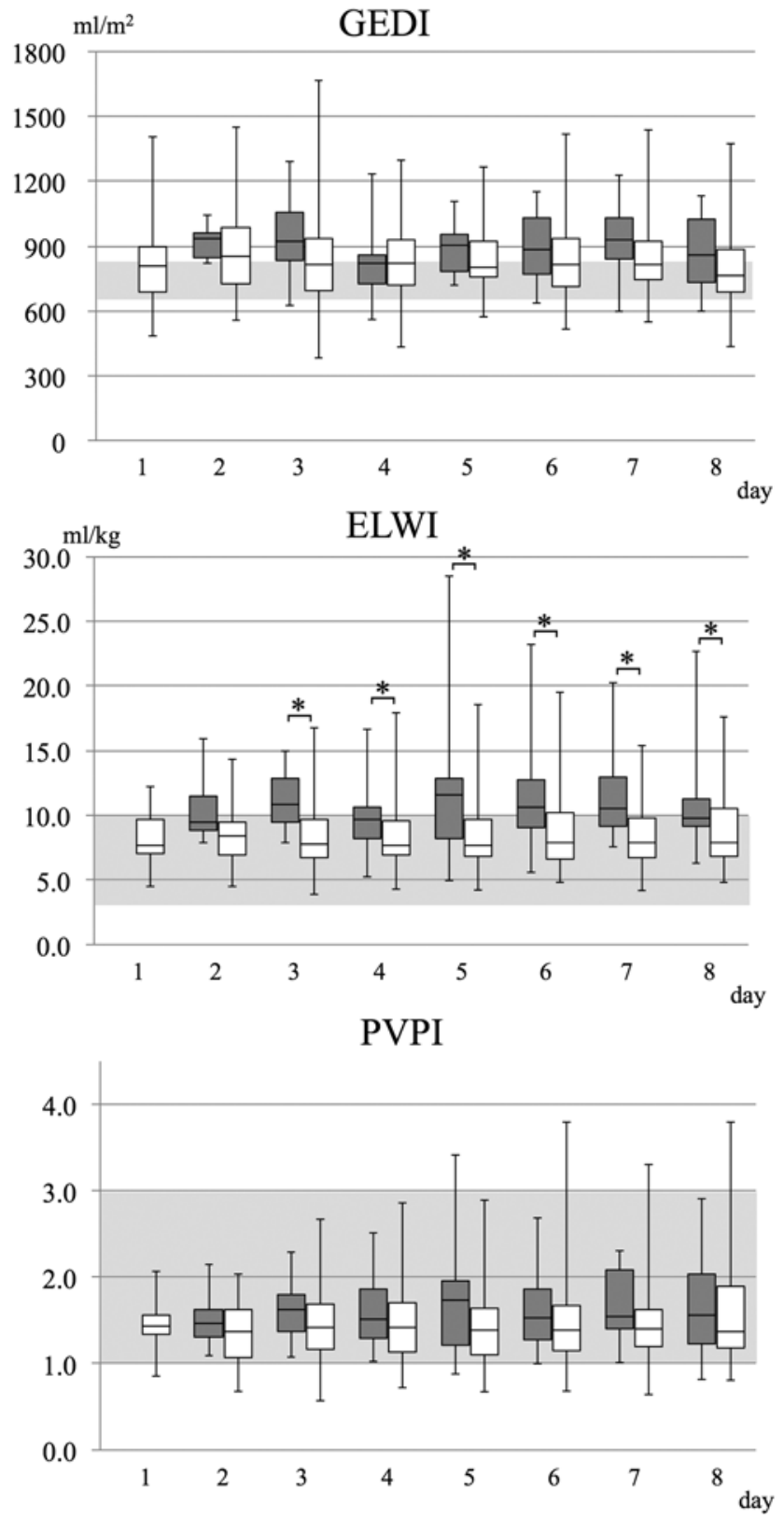

FIG. 4. PiCCO-plus monitoring in the early phase. Gray and white boxes represent data from the PE and non-PE groups, respectively; asterisks indicate statistical significance.

and aneurysm location did not differ significantly between the $\mathrm{PE}$ and non-PE groups. The incidence of pulmonary edema in patients who received triple-H therapy was $32 \%$, which is similar to that of the entire study population (25.5\%). Furthermore, triple-H therapy did not affect the development of pulmonary edema even after adjusting for circulatory data, including water balance. Moreover, Tagami et al. ${ }^{36}$ reported that triple-H therapy is not associated with improved clinical outcomes or quantitative hemodynamic indicators of intravascular volume.

The GEDI, CI, ELWI, and PVPI were examined to evaluate the cause of pulmonary edema and patient hemodynamics after SAH. According to the Frank-Starling 

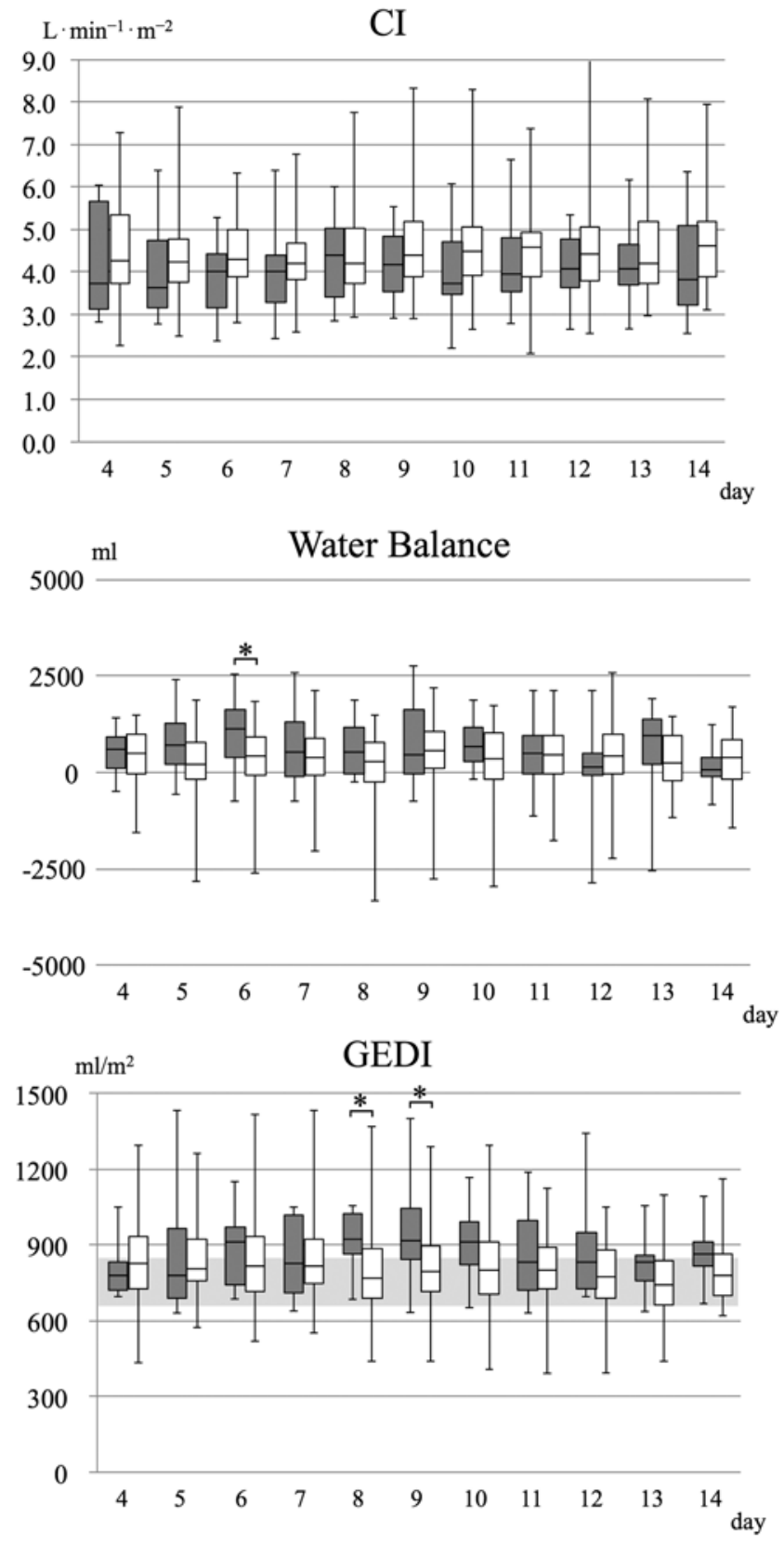

FIG. 5. PiCCO-plus monitoring in the delayed phase. Gray and white boxes represent data from the PE and non-PE groups, respectively; asterisks indicate statistical significance.

law, ${ }^{8}$ which describes the relationship between cardiac preload and the force of cardiac contraction, the force of left-ventricular contraction increases with increasing leftventricular end-diastolic volume. ${ }^{1}$ Thus, preload is described in terms of left-ventricular end-diastolic volume. PiCCO-plus monitoring assesses the GEDI according to transpulmonary thermodilution. The GEDI clearly indicates cardiac preload under all circumstances, including mechanical ventilation, and can serve as a predictive factor for DCI and pulmonary edema. Yoneda et al.$^{39}$ reported that patients with DCI exhibit a decreased GEDI in the early stage of SAH (i.e., Days 3-5). Meanwhile, Tagami
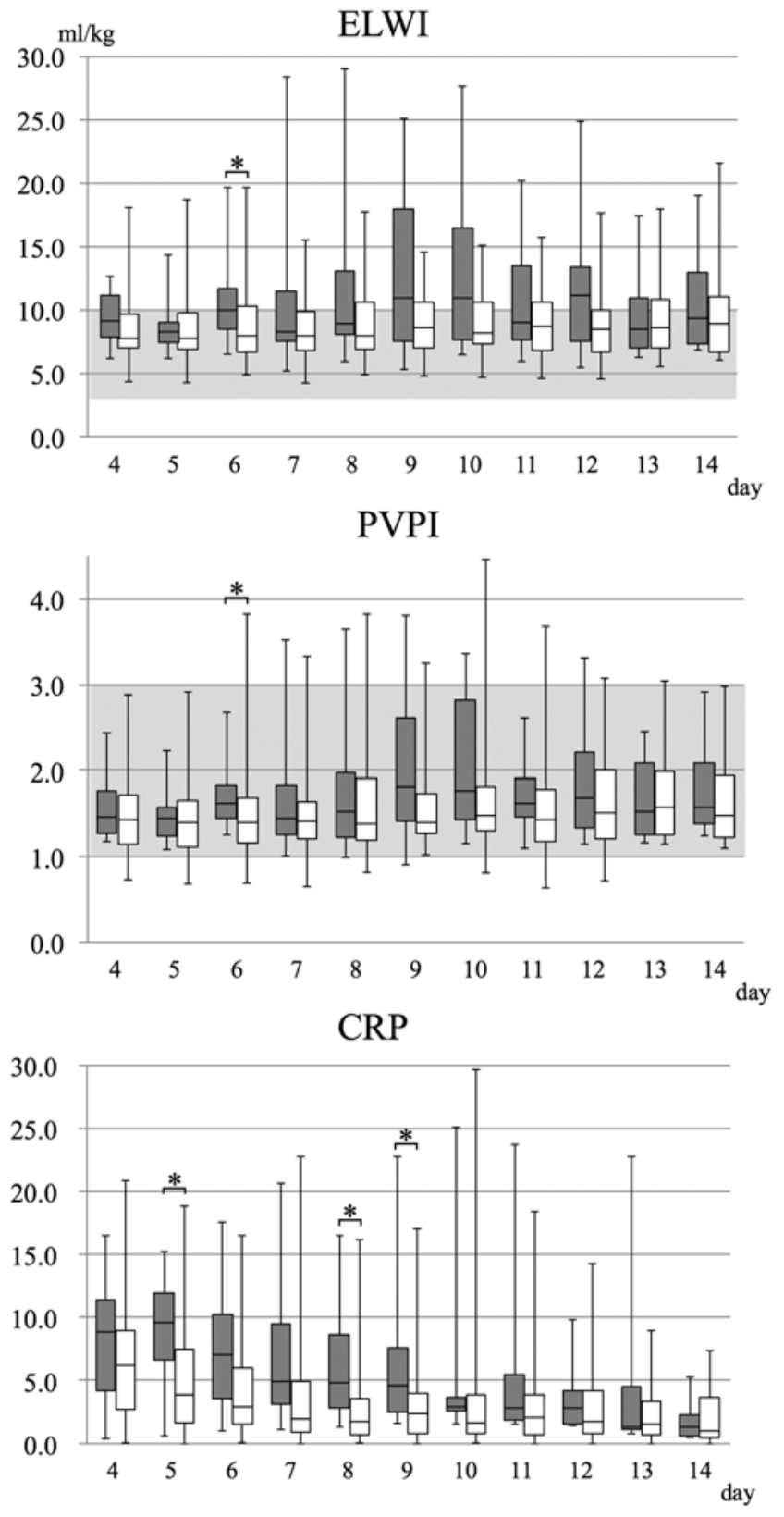

FIG. 6. PiCCO-plus monitoring in the delayed phase. Gray and white boxes represent data from the PE and non-PE groups, respectively; asterisks indicate statistical significance.

et al. ${ }^{35}$ demonstrated that the mean GEDI is an independent predictor of the occurrence of pulmonary edema, and a GEDI of $>921 \mathrm{ml} / \mathrm{m}^{2}$ is significantly associated with the development of severe pulmonary edema. In addition, an ELWI of $>10 \mathrm{ml} / \mathrm{kg}$ is more accurate for diagnosing pulmonary edema than clinical symptoms or chest radiograph findings. ${ }^{22}$ Furthermore, the ELWI is an independent predictor of prognosis. ${ }^{22}$ PVPI measurements can be used to differentiate hydrostatic pulmonary edema from acute lung injury/acute respiratory distress syndrome (i.e., noncardiogenic pulmonary edema) with acceptable accuracy. ${ }^{9,13,23}$ 

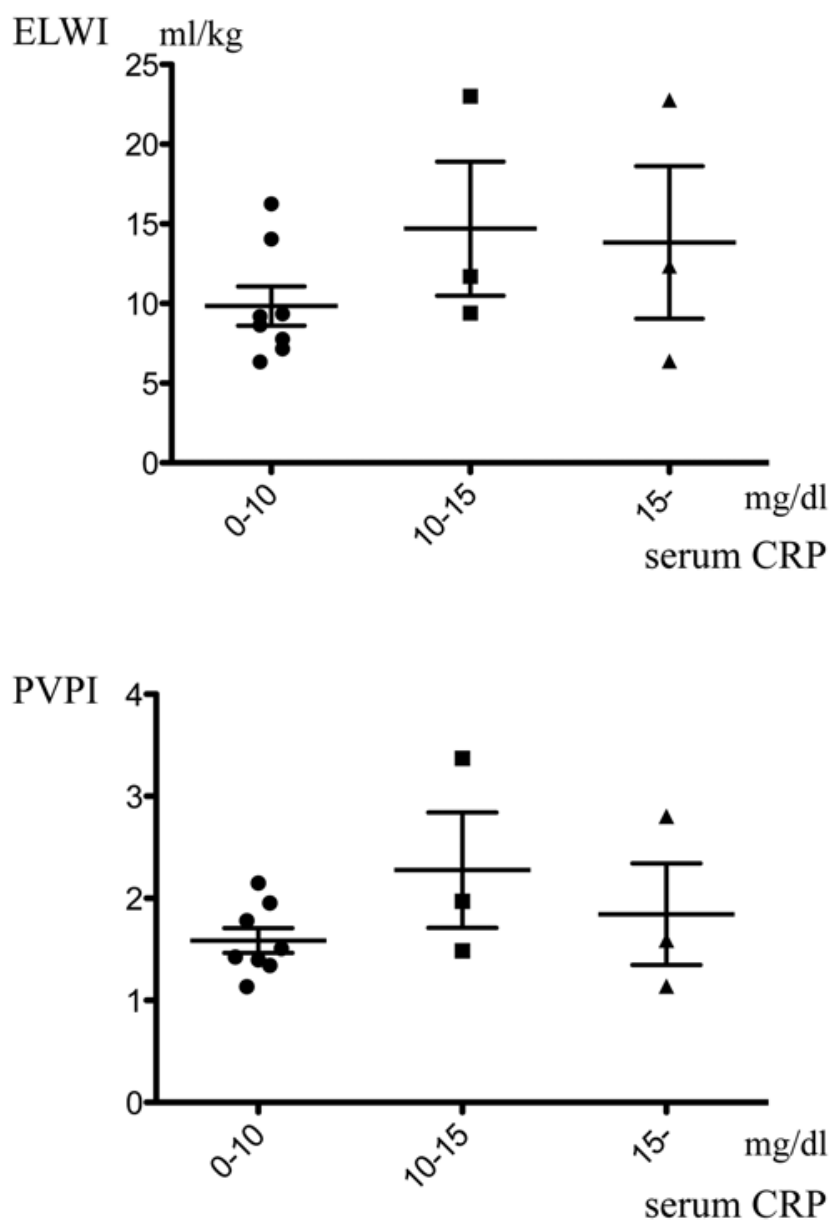

FIG. 7. ELWI (upper) and PVPI (lower) classified according to serum CRP levels in the PE group in the delayed phase. Serum CRP levels for each patient are plotted: $<10 \mathrm{mg} / \mathrm{dl}$ (circles), $10-15 \mathrm{mg} / \mathrm{dl}$ (squares), and $>15 \mathrm{mg} / \mathrm{dl}$ (triangles).

Pulmonary edema can be differentiated as cardiogenic or noncardiogenic. Cardiogenic pulmonary edema is caused by increased hydrostatic pressure secondary to increased pulmonary vascular pressure. ${ }^{23,33}$ Meanwhile, noncardiogenic pulmonary edema is caused by changes in the permeability of the pulmonary capillary membrane as a result of a direct or indirect pathological process. ${ }^{23}$ Several conditions (such as aspiration) directly injure the lungs and alveoli, whereas systemic conditions (such as severe sepsis and severe pancreatitis) damage the lungs via indirect mechanisms. ${ }^{29}$ Neurogenic pulmonary edema is a noncardiogenic pulmonary edema that develops during the acute phase of SAH. This clinical syndrome is characterized by an increase in interstitial and alveolar fluid in response to an acute central nervous system injury (e.g., SAH or severe head injury). ${ }^{4}$ Neurogenic pulmonary edema occurs as a result of a surge in the levels of endogenous serum catecholamines, which may result in changes in cardiopulmonary hemodynamics. ${ }^{3}$

We evaluated patient hemodynamics after SAH by using a PiCCO-plus device and found that the cause of pulmonary edema differed according to the timing of its
TABLE 2. ORs for pulmonary edema in the delayed phase

\begin{tabular}{lccl}
\hline \multicolumn{1}{c}{ Variable } & Adjusted OR & $95 \% \mathrm{Cl}$ & p Value \\
\hline Age & 1.26 & $1.07-1.66$ & 0.0024 \\
\hline M sex & 3.38 & $0.044-618.4$ & 0.570 \\
\hline WFNS grade & 18.51 & $1.78-1648$ & 0.009 \\
\hline Fisher grade & 3.44 & $0.25-164.9$ & 0.373 \\
\hline Triple-H therapy & 0.23 & $0.0016-7.16$ & 0.422 \\
\hline CRP (Day 6) & 0.83 & $0.32-2.04$ & 0.654 \\
\hline CRP (Day 7) & 1.13 & $0.48-2.47$ & 0.750 \\
\hline ELWI (Day 6) & 0.56 & $0.090-1.94$ & 0.383 \\
\hline ELWI (Day 7) & 1.49 & $0.41-8.40$ & 0.577 \\
\hline PVPI (Day 6) & 57,719 & 2.93 to 3.40 $\times 10^{11}$ & 0.024 \\
\hline PVPI (Day 7) & 0.005 & $0.004-15.9$ & 0.267 \\
\hline
\end{tabular}

onset. According to the PiCCO databank, 19 patients developed pulmonary edema on the day of SAH onset. In these cases, pulmonary edema was caused specifically by neurogenic pulmonary edema. In the other cases of pulmonary edema, a significant biphasic decrease in the CI was observed. The daily water balance of the PE group was slightly positive in the early phase. The PVPI was significantly higher in the PE group than in the non-PE group at 2 separate time points. Sato et al..$^{32}$ reported that the onset of pulmonary edema after $\mathrm{SAH}$ is biphasic. ${ }^{32} \mathrm{PE}$ that occurs immediately after SAH is cardiogenic because of low contractility, whereas PE that occurs on Day 7 or later after SAH is hydrostatic because of low contractility and hypervolemia. In our study, although the onset of pulmonary edema after SAH was biphasic, the mechanisms of pulmonary edema clearly differed between the early and delayed phases. In particular, low contractility was not observed in the delayed phase, which was attributed to the improved management of SAH with the PiCCO-plus device.

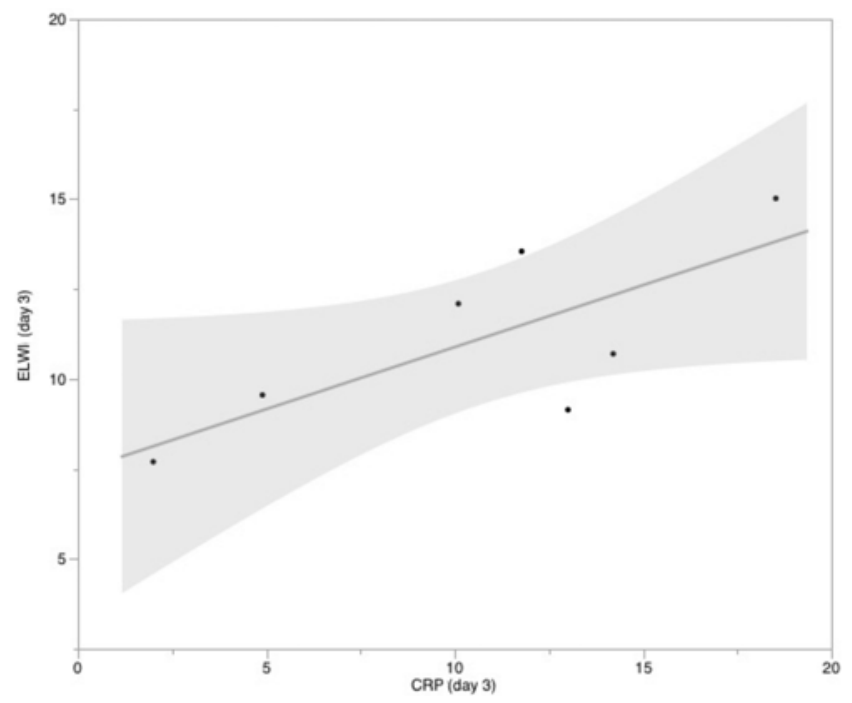

FIG. 8. Linear regression curves for the correlation between the serum CRP level and ELWI on Day 3 in the delayed-phase PE group $(r=0.61$, Spearman's rank correlation coefficient). 
In the PE group, the CI decreased without any change in the SVRI in the early phase. During this period, net water balance was positive, whereas the GEDI, which is a preload indicator, increased slightly. Meanwhile, the PVPI did not increase, and serum brain natriuretic peptide levels were higher. During the early phase, the numbers of patients with preexisting conditions that affect cardiac function were not significantly different between the PE and non-PE groups. These findings indicate that pulmonary edema that occurs in the early phase after SAH is a result of cardiac failure.

Changes in cardiac function were not observed during the delayed phase. However, net water balance was positive on Day 6, and preload increased significantly on Days 8 and 9. Therefore, pulmonary edema that occurs in the delayed phase is noncardiogenic pulmonary edema with hypervolemia. It was difficult to determine whether a direct or indirect mechanism was involved in the etiology of noncardiogenic pulmonary edema. Thus, the cause of noncardiogenic pulmonary edema was attributed to an inflammatory process. Multivariate analysis revealed that the high PVPI on Day 6 was independently associated with the occurrence of pulmonary edema in the delayed phase. Although PiCCO parameters were not associated with the serum CRP level, the high serum CRP levels and PVPIs in the delayed-phase PE group suggest that pulmonary edema in the delayed phase is caused by inflammation. The serum CRP level on Day 3 indicated that the general inflammatory reaction in the early phase may be useful in predicting the occurrence of pulmonary edema in the delayed phase. Thus, patients with a serum CRP level of $>$ $7.4 \mathrm{mg} / \mathrm{dl}$ on Day 3 after SAH may be at an increased risk of developing pulmonary edema in the delayed phase.

The limitations of this study include the lack of PiCCO data at the time of SAH onset and the lack of cardiac function evaluation. Because neurogenic pulmonary edema occurred in the early phase, it was not evaluated by using PiCCO data. Second, echocardiographic and electrocardiographic data were not recorded in the PiCCO databank for the evaluation of cardiac function. The PiCCO data indicated that cardiogenic causes were responsible for pulmonary edema that occurred in the early phase after SAH. However, echocardiographic or electrocardiographic data are required to explain the cause of the cardiac dysfunction.

\section{Conclusions}

The results of this study have clarified the circulatory characteristics of pulmonary edema after SAH. The cause of pulmonary edema is related to the timing of its onset after SAH. In the early phase, cardiac failure is the cause of pulmonary edema. PiCCO-plus monitoring can be used to easily obtain the ELWI, CI, and PVPI, which are helpful in diagnosing pulmonary edema.

\section{Appendix}

\section{Participating SAH PiCCO Study Group Centers}

Department of Emergency and Critical Care Medicine, Nippon Medical School Hospital; Department of Neurosurgery, Kansai Medical University Hirakata Hospital; Department of Neurosur- gery, Tokyo Medical and Dental University, University Hospital of Medicine; Department of Neurosurgery, Fukuoka University Hospital; Department of Neurosurgery, Nagasaki University Hospital; Department of Neurosurgery and Clinical Neuroscience, Yamaguchi University School of Medicine; Department of Emergency and Critical Care Medicine, Nippon Medical School Tama Nagayama Hospital; Department of Neurosurgery, Saitama Medical University General Medical Center; and Department of Neurosurgery, Saitama Medical University International Medical Center.

\section{Acknowledgments}

We thank Motoki Inaji, MD, PhD (Department of Neurosurgery, Tokyo Medical and Dental University), for advice regarding the statistical analysis.

\section{References}

1. Ashrafian H, Williams L, Frenneaux MP: The pathophysiology of heart failure: a tale of two old paradigms revisited. Clin Med 8:192-197, 2008

2. Awad IA, Carter LP, Spetzler RF, Medina M, Williams FC Jr: Clinical vasospasm after subarachnoid hemorrhage: response to hypervolemic hemodilution and arterial hypertension. Stroke 18:365-372, 1987

3. Baumann A, Audibert G, McDonnell J, Mertes PM: Neurogenic pulmonary edema. Acta Anaesthesiol Scand 51:447455, 2007

4. Davison DL, Terek M, Chawla LS: Neurogenic pulmonary edema. Crit Care 16:212, 2012

5. Egge A, Waterloo K, Sjøholm H, Solberg T, Ingebrigtsen T, Romner B: Prophylactic hyperdynamic postoperative fluid therapy after aneurysmal subarachnoid hemorrhage: a clinical, prospective, randomized, controlled study. Neurosurgery 49:593-606, 2001

6. Fisher CM, Kistler JP, Davis JM: Relation of cerebral vasospasm to subarachnoid hemorrhage visualized by computerized tomographic scanning. Neurosurgery 6:1-9, 1980

7. Friedman JA, Pichelmann MA, Piepgras DG, McIver JI, Toussaint LG III, McClelland RL, et al: Pulmonary complications of aneurysmal subarachnoid hemorrhage. Neurosurgery 52:1025-1032, 2003

8. Glower DD, Spratt JA, Snow ND, Kabas JS, Davis JW, Olsen $\mathrm{CO}$, et al: Linearity of the Frank-Starling relationship in the intact heart: the concept of preload recruitable stroke work. Circulation 71:994-1009, 1985

9. Groeneveld AB, Verheij J: Extravascular lung water to blood volume ratios as measures of permeability in sepsis-induced ALI/ARDS. Intensive Care Med 32:1315-1321, 2006

10. Gruber A, Reinprecht A, Görzer H, Fridrich P, Czech T, Illievich UM, et al: Pulmonary function and radiographic abnormalities related to neurological outcome after aneurysmal subarachnoid hemorrhage. J Neurosurg 88:28-37, 1998

11. Hoff RG, Rinkel GJ, Verweij BH, Algra A, Kalkman CJ: Pulmonary edema and blood volume after aneurysmal subarachnoid hemorrhage: a prospective observational study. Crit Care 14:R43, 2010

12. Hunt WE, Kosnik EJ: Timing and perioperative care in intracranial aneurysm surgery. Clin Neurosurg 21:79-89, 1974

13. Isakow W, Schuster DP: Extravascular lung water measurements and hemodynamic monitoring in the critically ill: bedside alternatives to the pulmonary artery catheter. Am J Physiol Lung Cell Mol Physiol 291:L1118-L1131, 2006

14. Jennett B, Bond M: Assessment of outcome after severe brain damage. Lancet 1:480-484, 1975

15. Kassell NF, Torner JC, Haley EC Jr, Jane JA, Adams HP, Kongable GL: The International Cooperative Study on the Timing of Aneurysm Surgery. Part 1: Overall management results. J Neurosurg 73:18-36, 1990 
16. Katayama Y, Haraoka J, Hirabayashi H, Kawamata T, Kawamoto K, Kitahara T, et al: A randomized controlled trial of hydrocortisone against hyponatremia in patients with aneurysmal subarachnoid hemorrhage. Stroke 38:2373-2375, 2007

17. Kosnik EJ, Hunt WE: Postoperative hypertension in the management of patients with intracranial arterial aneurysms. J Neurosurg 45:148-154, 1976

18. Kuroiwa T, Morita H, Tanabe H, Ohta T: Significance of ST segment elevation in electrocardiograms in patients with ruptured cerebral aneurysms. Acta Neurochir (Wien) 133:141146, 1995

19. Lennihan L, Mayer SA, Fink ME, Beckford A, Paik MC, Zhang H, et al: Effect of hypervolemic therapy on cerebral blood flow after subarachnoid hemorrhage: a randomized controlled trial. Stroke 31:383-391, 2000

20. Mayer SA, Fink ME, Homma S, Sherman D, LiMandri G, Lennihan L, et al: Cardiac injury associated with neurogenic pulmonary edema following subarachnoid hemorrhage. Neurology 44:815-820, 1994

21. Meier P, Zierler KL: On the theory of the indicator-dilution method for measurement of blood flow and volume. J Appl Physiol 6:731-744, 1954

22. Michard F: Bedside assessment of extravascular lung water by dilution methods: temptations and pitfalls. Crit Care Med 35:1186-1192, 2007

23. Monnet X, Anguel N, Osman D, Hamzaoui O, Richard C, Teboul JL: Assessing pulmonary permeability by transpulmonary thermodilution allows differentiation of hydrostatic pulmonary edema from ALI/ARDS. Intensive Care Med 33:448-453, 2007

24. Muroi C, Keller M, Pangalu A, Fortunati M, Yonekawa Y, Keller E: Neurogenic pulmonary edema in patients with subarachnoid hemorrhage. J Neurosurg Anesthesiol 20:188192,2008

25. Neumann P: Extravascular lung water and intrathoracic blood volume: double versus single indicator dilution technique. Intensive Care Med 25:216-219, 1999

26. Newman EV, Merrell M, Genecin A, Monge C, Milnor WR, McKeever WP: The dye dilution method for describing the central circulation. An analysis of factors shaping the timeconcentration curves. Circulation 4:735-746, 1951

27. Horie N, Isotani E, Honda S, Oshige H, Nagata I: Impact of aneurysm location on cardiopulmonary dysfunction after subarachnoid hemorrhage. J Stroke Cerebrovasc Dis 23:1795-1804, 2014

28. Horie N, Iwaasa M, Isotani E, Ishizaka S, Inoue T, Nagata I: Impact of clipping versus coiling on postoperative hemodynamics and pulmonary edema after subarachnoid hemorrhage. BioMed Res Int 2014:807064, 2014

29. Perina DG: Noncardiogenic pulmonary edema. Emerg Med Clin North Am 21:385-393, 2003

30. Sakka SG, Klein M, Reinhart K, Meier-Hellmann A: Prognostic value of extravascular lung water in critically ill patients. Chest 122:2080-2086, 2002

31. Sakka SG, Rühl CC, Pfeiffer UJ, Beale R, McLuckie A, Re- inhart K, et al: Assessment of cardiac preload and extravascular lung water by single transpulmonary thermodilution. Intensive Care Med 26:180-187, 2000

32. Sato Y, Isotani E, Kubota Y, Otomo Y, Ohno K: Circulatory characteristics of normovolemia and normotension therapy after subarachnoid hemorrhage, focusing on pulmonary edema. Acta Neurochir (Wien) 154:2195-2202, 2012

33. Smith WS, Matthay MA: Evidence for a hydrostatic mechanism in human neurogenic pulmonary edema. Chest 111:1326-1333, 1997

34. Suarez JI, Qureshi AI, Yahia AB, Parekh PD, Tamargo RJ, Williams MA, et al: Symptomatic vasospasm diagnosis after subarachnoid hemorrhage: evaluation of transcranial Doppler ultrasound and cerebral angiography as related to compromised vascular distribution. Crit Care Med 30:1348-1355, 2002

35. Tagami T, Kuwamoto K, Watanabe A, Unemoto K, Yokobori S, Matsumoto G, et al: Optimal range of global end-diastolic volume for fluid management after aneurysmal subarachnoid hemorrhage: a multicenter prospective cohort study. Crit Care Med 42:1348-1356, 2014

36. Tagami T, Kuwamoto K, Watanabe A, Unemoto K, Yokobori $\mathrm{S}$, Matsumoto $\mathrm{G}$, et al: Effect of triple-H prophylaxis on global end-diastolic volume and clinical outcomes in patients with aneurysmal subarachnoid hemorrhage. Neurocrit Care 21:462-469, 2014

37. Teasdale GM, Drake CG, Hunt W, Kassell N, Sano K, Pertuiset B, et al: A universal subarachnoid hemorrhage scale: report of a committee of the World Federation of Neurosurgical Societies. J Neurol Neurosurg Psychiatry 51:1457, 1988

38. Wood JH, Fleischer AS: Observations during hypervolemic hemodilution of patients with acute focal cerebral ischemia. JAMA 248:2999-2304, 1982

39. Yoneda H, Nakamura T, Shirao S, Tanaka N, Ishihara H, Suehiro E, et al: Multicenter prospective cohort study on volume management after subarachnoid hemorrhage: hemodynamic changes according to severity of subarachnoid hemorrhage and cerebral vasospasm. Stroke 44:2155-2161, 2013

\section{Disclosures}

The authors report no conflict of interest concerning the materials or methods used in this study or the findings specified in this paper.

\section{Author Contributions}

Acquisition of data: Takeda, Sato, Ishikura, Matsui, Isotani. Analysis and interpretation of data: Obata, Isotani. Drafting the article: Obata. Reviewed submitted version of manuscript: Isotani. Administrative/technical/material support: Takeda. Study supervision: Isotani.

\section{Correspondence}

Yoshiki Obata, Department of Neurosurgery, Tokyo Medical and Dental University, 1-5-45, Yushima, Bunkyo-ku, Tokyo 1138519, Japan. email: obata-tmd@umin.ac.jp. 